\title{
Modified management mode for colorectal cancer during COVID-19 outbreak - a single-center experience
}

\author{
Dexiang Zhu ${ }^{1,},{ }^{*}, \mathrm{Wu}^{1}{ }^{1,}, \mathrm{Qi} \mathrm{Lin}^{1}, \mathrm{Ye} \mathrm{Wei}^{1}$ \\ ${ }^{1}$ Department of General Surgery, Zhongshan Hospital, Fudan University, Shanghai, China \\ *Equal contribution
}

Correspondence to: Qi Lin, Ye Wei; email: lin777qi@163.com, 13818661815@126.com

Keywords: COVID-19, colorectal cancer, management model

Received: February 25, 2020 Accepted: April 4, 2020

Published: May 5, 2020

Copyright: Zhu et al. This is an open-access article distributed under the terms of the Creative Commons Attribution License (CC BY 3.0), which permits unrestricted use, distribution, and reproduction in any medium, provided the original author and source are credited.

\begin{abstract}
During the epidemic of COVID-19, the management model of colorectal cancer has to be changed at our center due to relatively limited medical resources. Outpatient visits are reduced under well protected after appointment, and rigorous investigation of epidemiological history and clinical symptoms are needed. We prefer a simple and convenient treatment regimen, which may also be postponed appropriately. Minimally invasive CRC surgery combined with a perioperative program of enhanced recovery after surgery should be recommended. We also focus on mental health treatments and healthy lifestyle education. In addition, routine follow-up can be moderately delayed. In total, adequate doctor-patient communication is also recommended throughout the treatment.
\end{abstract}

\section{INTRODUCTION}

From the end of 2019, a war without gunpowder has begun in China. Novel coronavirus pneumonia (COVID-19) from Wuhan city has now spread to the whole country and even the world [1]. The major routes of the coronavirus infection are the respiratory droplets, close contact transmission, and also when exposed to high concentrations of aerosol in a relatively closed environment for a long time [2]. The incubation period of COVID-19 is up to 24 days. The most common symptoms were fever and cough, and some severe cases can quickly progress to acute respiratory distress syndrome [3]. The Chinese government has initiated a first-level response to major public health emergencies, mobilized the whole country to fight against the epidemic, made comprehensive deployments, and implemented the strongest and strictest prevention and control measures. By the end of March, the epidemic situation has been under control across China. However, the pandemic of COVID-19 is global now.

Colorectal cancer (CRC) is the malignancy with the fourth highest prevalence among females and fifth among males in China [4]. CRC patients were generally in poor immunity and physical fitness, which are susceptible to COVID-19. A prospective cohort study has found that cancer patients were at higher risk of COVID-19 infection and had a worse prognosis than those without tumors [5].

During this particular period, most hospitals have suspended or postponed outpatient and elective surgery. Therefore, how to deal with CRC patients is challenging and essential. As follows, we introduce our single center's experience in the management of CRC patients during COVID-19 outbreak and present a series of issues of our clinical work (Table 1).

\section{Outpatient}

If CRC patients have obvious symptoms of bleeding, perforation, obstruction, or extreme discomfort, we recommend them to go to the emergency as soon as possible. Regular outpatient visits can be postponed appropriately. With the joint efforts of the whole country, the epidemic situation has changed positively, and outpatient in various places has gradually restored. 
Table 1. Changes in clinical practice during COVID-19 outbreak.

\begin{tabular}{|c|c|c|}
\hline Terms & & Details \\
\hline \multirow[t]{5}{*}{ Outpatient } & Appointment & Online \\
\hline & Escort & One at most \\
\hline & Protection & A medical surgical mask or a general medical mask at least \\
\hline & Screening & $\begin{array}{l}\text { Check body temperature; Check for signs and symptoms; Epidemiological } \\
\text { investigation }\end{array}$ \\
\hline & Treatment & Reduce infusion time; Long prescription policy \\
\hline & Neoadjuvant therapy & $\begin{array}{l}\text { Prefer neoadjuvant chemotherapy alone; Expand neoadjuvant therapy } \\
\text { indications for low to moderate locally advanced rectal cancer }\end{array}$ \\
\hline & mCRC patient & $\begin{array}{l}\text { Develop, improve or change the regimen by online MDT clinics or Wechat } \\
\text { group }\end{array}$ \\
\hline & Traditional Chinese Medicine & Follow the physicians' advice \\
\hline Daily life & & Mental health; Healthy lifestyle \\
\hline Follow up & & $\begin{array}{l}\text { Postpone review time appropriately; The principle of proximity hospital; } \\
\text { Online follow-up }\end{array}$ \\
\hline
\end{tabular}

\section{Appointment}

Not as the previous, all outpatient clinics request appointments now. CRC patients should make an appointment in advance, and only one family member is allowed to accompany to prevent cross-infection.

\section{Protection}

The medical workers, patients and families need to strengthen their own protection, such as wearing surgical masks, goggles, and so on. We also pay attention to the disinfection of outpatient equipment and the environment.

\section{Epidemiological history}

At the clinic, a rigorous investigation of epidemiological history and clinical symptoms is needed. Patients with a history of living or traveling in the affected area, close contacts with a confirmed or probable case, or having fever and respiratory symptoms, should be checked during pre-diagnosis. And then, if suspected COVID-19 manifestations, the patient should be sent to the fever clinic. If a suspected or confirmed case is diagnosed, the patient shall be immediately quarantined and reported.

\section{Outpatient treatment}

At the outpatient, we choose simple and convenient regimen, and also establish a long prescription policy to facilitate patients to receive drugs for 2 to 3 months at a time, to reduce the times of visiting.

\section{Non-surgical treatment}

The Chinese Society of Clinical Oncology guidelines recommend that adjuvant chemotherapy should be started as soon as possible after recovery, generally about 3 weeks after CRC operation, and no later than 2 months [6]. A meta-analysis of 15410 CRC patients showed that the start of postoperative adjuvant chemotherapy was delayed every 4 weeks, patients' overall survival time and disease-free survival time will be significantly reduced [7]. Therefore, we recommend postponing adjuvant chemotherapy appropriately at the local hospital as the first choice. Moreover, we prefer the three-week CapeOX regimen to biweekly FOLFOX regimen, so that we can minimize the chance of cross-infection. Oral capecitabine monotherapy also could be used as much as possible. In addition, we recommend that the patients can contact the physicians to reduce the treatment intensity and switch to oral therapy.

During the epidemic, many hospitals suspended radiotherapy. The Chinese FOWARC Trial showed that no significant difference in outcomes was found between mFOLFOX6 without radiotherapy and fluorouracil with radiotherapy for locally advanced rectal cancer [8]. Therefore, neoadjuvant chemotherapy alone with the mFOLFOX6 regimen is also an option. 
For patients with metastatic CRC, Multi-Disciplinary Treatment (MDT) is the best choice. If the condition is stable, the original chemotherapy regimen can be maintained for another 1-2 cycles until the MDT outpatient restore. If there is obvious progression, we recommend online MDT clinics or communicating with physicians via WeChat or telephone to change the regimen.

Undoubtedly, traditional Chinese medicine has certain effects on improving the physical condition of CRC patients, which can reduce the side effects of chemotherapy and improve the quality of life [9]. Considering the patients' resistance during chemotherapy is relatively low, we also recommend regular thymosin to improve immunity as prescribed [10].

\section{Surgery}

CRC surgeons should control the routine operation to reduce the patient's exposure time in the hospital [11]. For CRC patients with mass bleeding, perforation, or obstruction, emergency surgery should be considered, and COVID-19 infection needs to be ruled out before.

Endoscopic surgery is recommended for early-stage $\mathrm{CRC}$ when it is completely removed clearly with good histological features, and no additional surgical treatment is required. Whether surgery delay affects survival remains controversial for advanced CRC. We prefer to expand neoadjuvant therapy indications for low to moderate locally advanced rectal cancer. And we try to conduct surgery for advanced colon cancer as early as possible. A detailed investigation and a comprehensive examination (chest $\mathrm{CT}$ or viral nucleic acid test) should be performed to exclude COVID-19 before elective surgery. In addition, we also do not recommend to perform colorectal and liver resections simultaneously at the current situation, so as to avoid a prolonged hospital stay and increased risk of infection.

At the surgical ward, we prefer minimally invasive surgery plus a perioperative program of enhanced recovery after surgery (ERAS) as the best treatment strategy, which could accelerate patient recovery and shorten hospital stay [12].

The hospital should strictly implement the National Health Commission's requirements for infection control in medical institutions [13]. Ordinary patients who underwent $\mathrm{CRC}$ resection can be transferred to the general ward after surgery. It is necessary to reduce the movement of accompanying staff and personnel. Patients with postoperative fever should be carefully identified and isolated according to the suspected COVID-19 criteria [2]. And then suspected or confirmed patients should be transferred to a designated negative pressure isolation monitoring room for single room isolation.

\section{Daily life}

CRC and COVID-19 are double blows to patients. Many patients have mental health problems of anxiety and depression, so we should give positive psychological support during the epidemic. We inform that the prognosis of CRC is not so bad, and even with recurrence, a considerable part of the patients will be cured when metastases are detected and resected early [14]. Stoma patients are also encouraged to communicate with families and friends, learn to selfregulate bad moods, and actively integrate into society. At the same time, we also inform the patients that they will not be infected if actively protect, and the epidemic situation is getting better now, which will return to normal soon. Even if the treatment is appropriately delayed, it will not affect the treatment effect. At present, several public institutions and domestic hospitals have launched psychological hotline services, and we recommend patients could contact when needed.

A healthy lifestyle is especially important for CRC patients. Studies have shown that smokers have a significantly increased risk of developing and dying from CRC compared with never-smokers, and heavy drinking also increases the risk of developing CRC [15]. We recommend CRC patients a healthy and balanced lifestyle diet, avoiding high fat and low fiber diet, reducing the intake of red meat and processed meat. The American Gastroenterological Association has recommended calcium supplements for the primary or secondary prevention of colon cancer, so we also recommend appropriate intake of calcium-rich food such as dairy products [16]. CRC patients also need to appropriately increase the intake of cellulose and decrease irritating and too much oily food. Stoma patients can properly consume dairy products and vegetables to reduce the odor at the stoma. In addition, we also suggest that during the epidemic, patients can arrange indoor physical exercise under the guidance of the physicians, avoiding prolonged bed rest, which can promote the recovery of intestinal function and prevent deep vein thrombosis.

Recently, many health organizers have opened publicinterest online lectures and free mobile applications for different patient groups, to provide disease education and answer questions online. Furthermore, several university hospitals have also opened up various online clinics, including online fever clinics, psychological clinics, specialist clinics and online MDT clinics. In addition, we also have established several follow-up 
WeChat groups for CRC patients. When CRC patients have questions, they can get medical advice quickly from multiple experts at home.

\section{Follow up}

For patients who need to be reviewed after CRC surgery during the epidemic, we recommend that the review time can be appropriately postponed. We encourage patients to complete routine review projects at the nearest medical institution. After they obtain the review results, it is recommended to adopt an online network method for consultation.

In summary, our current clinical work model has to be changed due to COVID-19 outbreak, which includes the above outpatient, inpatient, psychological treatment and health education.

\section{CONFLICTS OF INTEREST}

The authors declare no conflicts of interest.

\section{FUNDING}

National Key Research and Development Program of China (2017YFC0908200); National Natural Science Foundation of China Youth Fund(81602036).

\section{REFERENCES}

1. Zhu N, Zhang D, Wang W, Li X, Yang B, Song J, Zhao X, Huang B, Shi W, Lu R, Niu P, Zhan F, Ma X, et al, and China Novel Coronavirus Investigating and Research Team. A Novel Coronavirus from Patients with Pneumonia in China, 2019. N Engl J Med. 2020; 382:727-33.

https://doi.org/10.1056/NEJMoa2001017

PMID:31978945

2. National Health Committee of the People's Republic of China. Diagnosis and treatment of novel coronavirus pneumonia (Pilot Sixth Edition). 2020 (in Chinese).

3. Guan WJ, Ni ZY, Hu Y, Liang WH, Ou CQ, He JX, Liu L, Shan H, Lei CL, Hui DSC, Du B, Li LJ, Zeng G, et al; China Medical Treatment Expert Group for Covid-19. Clinical Characteristics of Coronavirus Disease 2019 in China. N Engl J Med. 2020; 382:1708-20.

https://doi.org/10.1056/NEJMoa2002032

PMID:32109013

4. Chen W, Zheng R, Baade PD, Zhang S, Zeng H, Bray F, Jemal A, Yu XQ, He J. Cancer statistics in China, 2015. CA Cancer J Clin. 2016; 66:115-32. https://doi.org/10.3322/caac.21338 PMID:26808342
5. Liang W, Guan W, Chen R, Wang W, Li J, Xu K, Li C, Ai $\mathrm{Q}$, Lu W, Liang $\mathrm{H}$, Li S, He J. Cancer patients in SARSCoV-2 infection: a nationwide analysis in China. Lancet Oncol. 2020; 21:335-37.

https://doi.org/10.1016/S1470-2045(20)30096-6 PMID:32066541

6. Diagnosis And Treatment Guidelines For Colorectal Cancer Working Group CSOCOC. Chinese Society of Clinical Oncology (CSCO) diagnosis and treatment guidelines for colorectal cancer 2018 (English version). Chin J Cancer Res. 2019; 31:117-34.

https://doi.org/10.21147/j.issn.1000-9604.2019.01.07 PMID:30996570

7. Biagi JJ, Raphael MJ, Mackillop WJ, Kong W, King WD, Booth CM. Association between time to initiation of adjuvant chemotherapy and survival in colorectal cancer: a systematic review and meta-analysis. JAMA. 2011; 305:2335-42.

https://doi.org/10.1001/jama.2011.749

PMID:21642686

8. Deng $Y$, Chi $P$, Lan $P$, Wang L, Chen W, Cui L, Chen D, Cao J, Wei H, Peng X, Huang Z, Cai G, Zhao R, et al. Neoadjuvant Modified FOLFOX6 With or Without Radiation Versus Fluorouracil Plus Radiation for Locally Advanced Rectal Cancer: Final Results of the Chinese FOWARC Trial. J Clin Oncol. 2019; 37:3223-33.

https://doi.org/10.1200/JCO.18.02309 PMID:31557064

9. Tan KY, Liu CB, Chen AH, Ding YJ, Jin HY, SeowChoen $\mathrm{F}$. The role of traditional Chinese medicine in colorectal cancer treatment. Tech Coloproctol. 2008; 12:1-6. https://doi.org/10.1007/s10151-008-0392-z PMID:18512006

10. Xu Jian ZZ, Xing X. Effect of perioperative application of thymopentin on immune function in patients with rectal cancer. Chinese Journal of Bases and Clinics in General Surgery. 2016; 23:561-64.

11. Chinese Society of Colorectal Surgery, Chinese Society of Surgery, Chinese Medical Association; Colorectal and Anal Surgery Committee, Chinese Research Hospitals Association. Chinese expert consensus on surgical diagnosis and treatment strategies for colorectal cancer patients during novel coronavirus peneumonia epidemic. Chin J Pract Surg. 2020; 40:241-4 (in Chinese).

12. Vlug MS, Wind J, Hollmann MW, Ubbink DT, Cense HA, Engel AF, Gerhards MF, van Wagensveld BA, van der Zaag ES, van Geloven AA, Sprangers MA, Cuesta MA, Bemelman WA, and LAFA study group. Laparoscopy in combination with fast track multimodal management is the best perioperative strategy in patients undergoing colonic surgery: a randomized clinical trial (LAFA-study). Ann Surg. 2011; 254:868-75. 
https://doi.org/10.1097/SLA.0b013e31821fd1ce PMID:21597360

13. National Health Committee of the People's Republic of China. Notice on Printing and Distributing New Guides for the Prevention and Control of New Coronavirus Infection in Medical Institutions (First Edition)[EB/OL]. (in Chinese).

14. Kopetz S, Chang GJ, Overman MJ, Eng C, Sargent DJ, Larson DW, Grothey A, Vauthey JN, Nagorney DM, McWilliams RR. Improved survival in metastatic colorectal cancer is associated with adoption of hepatic resection and improved chemotherapy. J Clin Oncol. 2009; 27:3677-83.

https://doi.org/10.1200/JCO.2008.20.5278

PMID:19470929
15. Giovannucci E, Rimm EB, Ascherio A, Stampfer MJ, Colditz GA, Willett WC. Alcohol, low-methionine-lowfolate diets, and risk of colon cancer in men. J Natl Cancer Inst. 1995; 87:265-73.

https://doi.org/10.1093/jnci/87.4.265

PMID: 7707417

16. Bond $\mathrm{JH}$, and Practice Parameters Committee of the American College of Gastroenterology. Polyp guideline: diagnosis, treatment, and surveillance for patients with colorectal polyps. Am J Gastroenterol. 2000; 95:3053-63.

https://doi.org/10.1111/j.1572-0241.2000.03434.x PMID:11095318 